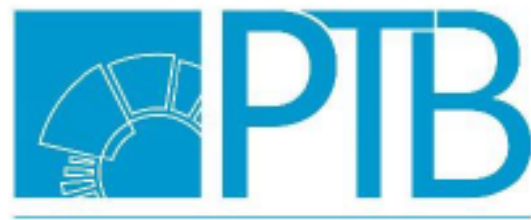

Physikalisch-Technische Bundesanstalt Braunschweig und Berlin

The following article is the final version submitted to IEEE after peer review; hosted by CIRCE; DOI: 10.1109/AMPS50177.2021.9586040. It is provided for personal use only.

\title{
Novel Calibration systems for the dynamic and steady-state testing of digital instrument transformers
}

\author{
Y. Chen, G. Crotti, A. Dubowik, P. S. Letizia, E. Mohns, M. Luiso, and J. Bruna
}

Acknowledgement: The presented work is used in the project 17IND06 (FutureGrid II) which have received funding from the EMPIR programme co-financed by the Participating States and from the European Union's Horizon 2020 research and innovation programme.

(c) 2018 IEEE. This is the author's version of an article that has been published by IEEE.

Personal use of this material is permitted. Permission from IEEE must be obtained for all other uses, in any current or future media, including reprinting/republishing this material for advertising or promotional purposes, creating new collective works, for resale or redistribution to servers or lists, or reuse of any copyrighted component of this work in other works.

Full Citation of the original article published be IEEE:

Y. Chen et al., "Novel Calibration systems for the dynamic and steady-state testing of digital instrument transformers," 2021 IEEE 11th International Workshop on Applied Measurements for Power Systems (AMPS), 2021, pp. 1-6, doi: 10.1109/AMPS50177.2021.9586040.

Available at: $\underline{\text { https://ieeexplore.ieee.org/document/9586040 }}$ 


\title{
Novel Calibration systems for the dynamic and steady-state testing of digital instrument transformers
}

\author{
Yeying Chen, Gabriella Crotti, Alexander Dubowik, Palma Sara Letizia, \\ Enrico Mohns, Mario Luiso, and Jorge Bruna
}

\begin{abstract}
Within the frame of the European project "Future Grid II - Metrology for the next-generation digital substation instrumentation", several partners developed traceable calibration systems which allow the calibration of conventional or nonconventional instrument transformers (IT) even with a sampled value (digital) output according to IEC 61869-9. Different setups are prepared to allow the calibration with complex test waveforms to emulate steady state, dynamic or temporary events during the assessment of the ITs. The laboratory calibration setups for either current transformers or voltage transformers are briefly described. Several results obtained for different kind of voltage or current transformers are presented.
\end{abstract}

Index Terms-Power quality, substation automation, smart grids, measurement techniques, instrument transformers.

\section{INTRODUCTION}

Standards for the assessment of the performance of Power Quality (PQ) and Phasor Measurement Unit (PMU) measuring instruments have been published in recent years. These standards include test scenarios that are believed to establish confidence in the measurement of real life events [1]-[5] However, these tests are performed without the required voltage and current transducers, which scale the high voltage and current to levels fitting into the analogue $\mathrm{PQ} / \mathrm{PMU}$ measuring instruments. Limited information on the effects introduced by analogue type Instrument Tranformer (IT) is available [6][8], while there is no scientific and technical knowledge about effects introduced by digital type IT. The behaviour of IT (both conventional and non-conventional) in the presence of most PQ phenomena is unknown, but essential for the interpretation of the $\mathrm{PQ} / \mathrm{PMU}$ measurement results.

In this scenario the European project "FUTURE GRID IImetrology for the next-generation digital substation instrumentation" aims to cover this knowledge gap by providing traceable characterization systems to allow the calibration of conventional or non-conventional instrument transformers for $\mathrm{PQ} / \mathrm{PMU}$ applications.

This work focuses on the new laboratory test systems for the IT characterisation up to $400 / \sqrt{3} \mathrm{kV}$ for voltage Low Power Instrument Tranformer (LPIT) and at least $2 \mathrm{kA}$ for

This project 17IND06 FutureGrid II has received funding from the EMPIR programme co-financed by the Participating States and from the European Union's Horizon 2020 research and innovation programme.

Y. Chen, A. Dubowik and E. Mohns are with Physikalisch-Technische Bundesanstalt (PTB), Germany. G. Crotti and P.S Letizia are with Istituto Nazionale di Ricerca Metrologica (INRIM), Italy. M. Luiso is with Università degli Studi della Campania "Luigi Vanvitelli", Italy. J. Bruna is with Centro de Investigación de Recursos y Consumos Energéticos (CIRCE Foundation), Spain (e-mail: jbruna@fcirce.es). current LPIT under steady-state (harmonics, interharmonics, etc.), dynamic (amplitude and phase modulation, frequency ramps, etc.) and temporary PQ phenomena (dips, swells, etc.). This is based on the identification and implementation of a set of reference test waveforms representative of real PQ phenomena. The basic uncertainty of the laboratory systems is in the order of $30 \mathrm{ppm}$ and $50 \mu \mathrm{rad}$, and the developed systems can generate and measure reference PQ events with an uncertainty from five times to one order of magnitude lower than the corresponding value for the class A compliant measuring instruments [1]. The relevant international standards that have been considered for this work can be divided into various categories, dealing with:

- instrument transformers;

- limits for disturbances in public Distribution Systems (DSs);

- measurement methods for PQ indices;

- test methods for PQ measuring instruments;

- test methods for synchrophasor measurement.

As a general comment, a considerable number of standards have been published on the PQ/PMU subject. On the other hand, the standards for ITs are focused on accuracy verification just at power frequency $(50 / 60 \mathrm{~Hz})$. Only the IEC $60044-$ 8 [9] and the IEC 61869-6 [10], for a small subset of ITs, deal also with the verification of accuracy at harmonic frequencies, but with signals of reduced amplitudes, without giving indications neither on possible setups to perform the tests nor providing methods for the uncertainty assessment. In this regard, this paper presents two novel generation and reference measuring systems for both current and voltage LPITs calibration. The ITs calibration systems introduced in this paper present several novelty features regarding the traditional setups for ITs characterization. Both the voltage and current generation systems allow to characterize ITs under current waveforms In fact, the generation systems can reproduce at Medium Voltage (MV) levels test waveforms representative of real PQ phenomena such as multitone signals, amplitude, and phase modulated signals, frequency ramps, dips, and swells. Moreover, to compare the analogue input of the digital type ITs with the Sampled Values (SVs) [11] at their output, the reference systems include accurate time synchronization sections. In this way, both the ITs input and output signals are referred to absolute time signals such as 1 PPS or Precise Time Protocol (PTP) $1588 \mathrm{v} 2$.

As regards the reference measurement chain, the reference voltage and current sensors and acquisitions systems are 
characterized by flat frequency responses over a wideband that is from $50 \mathrm{~Hz}$ up to $9 \mathrm{kHz}$.

The paper is organized as follows: Section II-A and Section III describe the calibration systems for current and voltage LPIT respectively. Section II-B, Section IV and Section V focus on description and results of tests performed on commercial LPIT with analog and digital output, whereas Section VI draws the conclusions.

\section{CAlibration SYSTEM FOR CURRENT LPITS}

\section{A. The calibration system and the generation}

The setup of a calibration system, more details in [12], for Current Transformer (CT) calibrations up to $2 \mathrm{kA}$, is shown in Fig. 1. The setup is mainly made up of a high current generation system (marked as red block), a set of analogue reference current transformers (marked as green block) with associated precision resistors and a precision two-channel measuring system (marked as purple block). The synchronization signals (remarked as "Sync." in blue) are necessary when the output of the Device Under Test (DUT) is a Sampled Value (SV) data stream. The synchronization signals can be obtained by Global Position System (GPS) receivers and be transmitted as $10 \mathrm{MHz}$, pulse per second (PPS) and IEEE 1588-2008 (PTPv2).

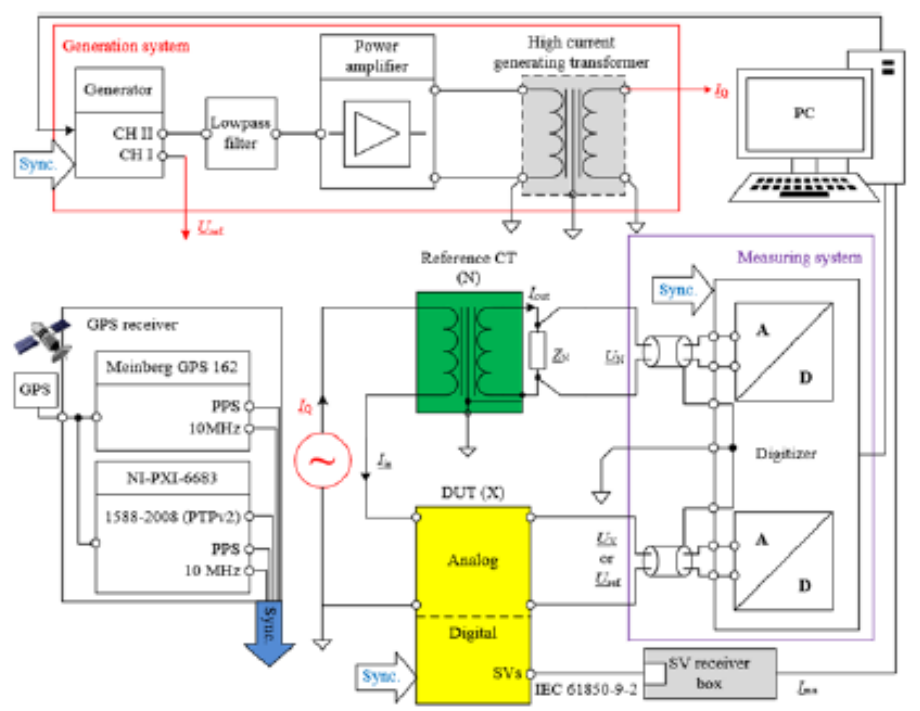

Fig. 1: Measurement setup for the current transformer calibrations.

In the following lines, each block will be described in detail to understand how the proposed calibration system for current LPIT was built.

For a digital instrument transformer, the ratio $F_{I, X}$ might be expressed by $\frac{I_{m u}}{I_{i n}}$ (or equivalently $\frac{U_{m u}}{U_{s n}}$ ). $I_{m u}$ represents the complex fundamental root mean square (rms) value, calculated from the discrete Fourier transform (DFT) of the sampled values. $I_{\text {in }}$ refers to the measured primary current by means of the reference current transformer $F_{I, N}$, the high accurate shunt $Z_{N}$ and the measured voltage $U_{N}$ of the digitiser. The measured complex primary current is determined according to:

$$
I_{i n}=\frac{U_{N}}{Z_{N} \times F_{I, N}}
$$

In order to relate the two measurements, the phase reference of the generator $U_{\text {ref }}$, which is synchronised to the PPS from the GPS receiver, is generated by a function generator as a sinusoidal signal without phase offset $\left(\arg \left(U_{r e f}\right)=0^{\circ}\right)$. For the digital instrument transformer calibration, $U_{\text {ref }}$ is connected to the Digitiser channel $U_{X}\left(U_{X}=U_{r e f}\right)$.

The complex voltage ratio $\Gamma_{X_{N}}=\frac{U_{X}}{U_{N}}$ is measured using a Digitizer:

$$
\Gamma_{X_{N}}=\frac{U_{X}}{U_{N}}=\frac{U_{r e f}}{I_{\text {in }} \times Z_{N} \times F_{I, N}}
$$

The ratio error $\varepsilon_{i}$ of the digital DUT is

$$
\varepsilon_{i}=\frac{I_{m u}}{I_{\text {in }}}-1
$$

The high current generation system, shown in Fig.1 marked as a red block, consists of a programmable 2-channel arbitrary waveform generator (more details in [12]), e.g. the Agilent $33500 \mathrm{~B}$ Series with resolution of 16 Bit shown in Fig.2, the lowpass filter, a transconductance power amplifier (up to 270 Vrms / 70 Arms, DC to $15 \mathrm{kHz}$ ), shown in Fig.3, and several high current generators rated from $100 \mathrm{~A}$ to $1 \mathrm{kA}$ and another one from 5 A to 200 A (Fig.4).

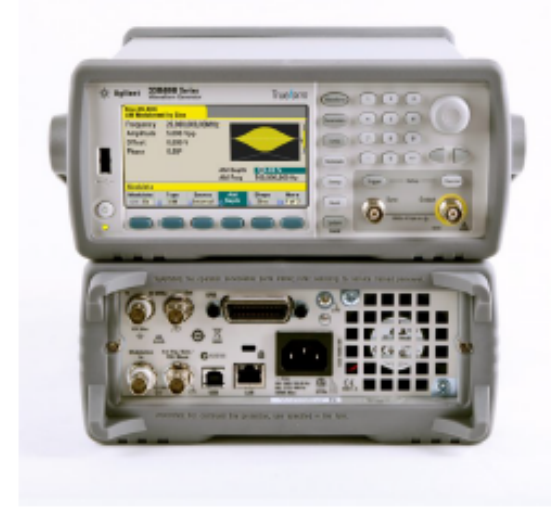

Fig. 2: Agilent 33522B.

\section{B. Experimental results}

The operation of the calibration system has been tested in preliminary measurements with three different kinds of current sensors. The first measurements have been done with three inductive CTs (class $0.2 \mathrm{~S}$, class 0.5 and class 1), see Fig.5. The measurements were executed with the primary current of $100 \mathrm{~A}$ in a frequency range from $50 \mathrm{~Hz}$ to $9 \mathrm{kHz}$. Measurement results can be seen in Fig.6.

Besides, three Rogowski coils from LEM were measured with three different diameters $(70 \mathrm{~mm}, 126 \mathrm{~mm}, 175 \mathrm{~mm})$ and with a rating of $1000 \mathrm{~A}, 22.5 \mathrm{mV}$ at $50 \mathrm{~Hz}$ (see Fig.7). In order to adapt the low output voltage to the input of the Digitiser, an electronic integrator has been built up. The gain and integration are chosen to obtain an output voltage of $250 \mathrm{mV}$ (range 1) and $2.75 \mathrm{~V}$ (range 2). The measurements were executed with the primary current of $100 \mathrm{~A}$ in a frequency range from $50 \mathrm{~Hz}$ 


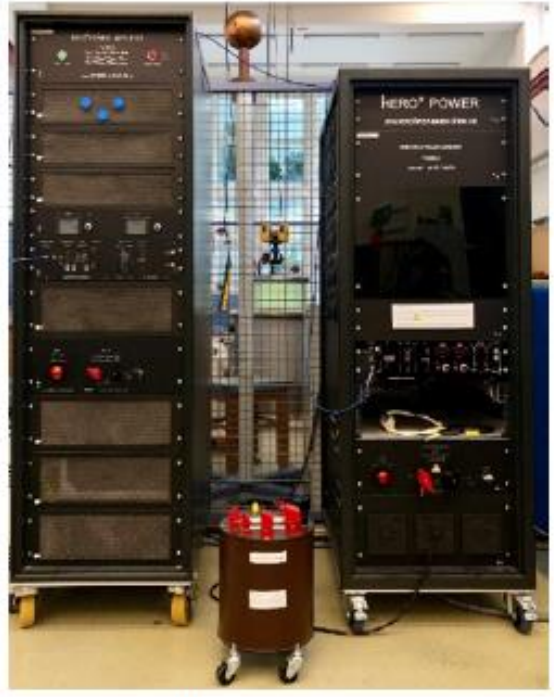

Fig. 3: Two power amplifiers and a current generation transformer with several rated current ranges from $5 \mathrm{~A}$ to $200 \mathrm{~A}$.

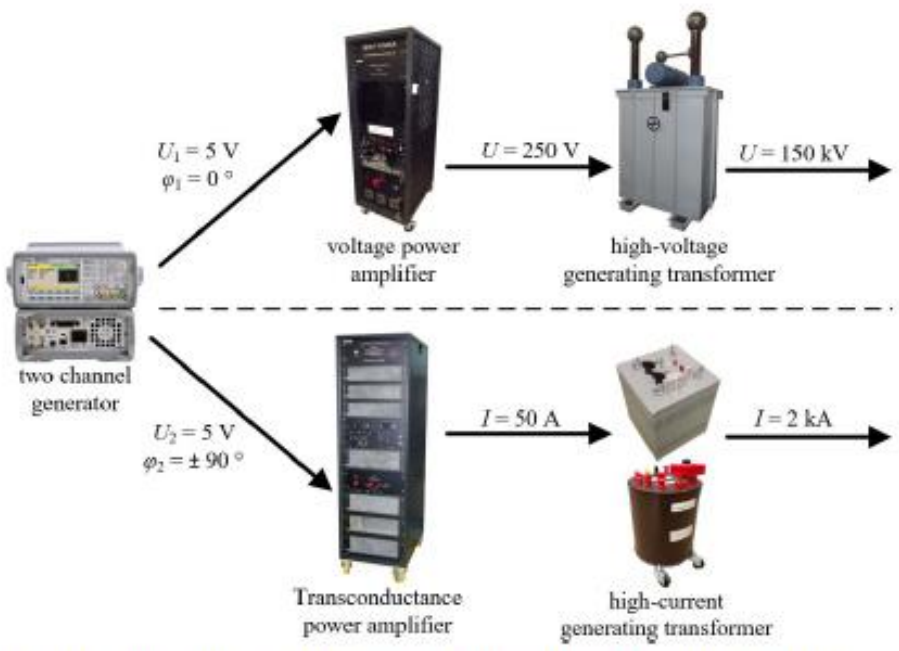

Fig. 4: Current generations and an option for a high voltage generation.

to $9 \mathrm{kHz}$. In the following figure it can be seen the obtained ratio errors and phase displacements after calibration.

Finally, one channel (L1) of a Siemens SAMU Siprotec 5 was measured by the rated input current of $1 \mathrm{~A}$ range (rated primary current as $1000 \mathrm{~A}$ ) with sampling rate of $4 \mathrm{kHz}$. The measurements were executed with the input currents ranging from $1 \%$ to $400 \%$ of $1 \mathrm{~A}$ at power frequency. Results can be seen on Table I.

\section{CAlibRation SYSTEM FOR VOLTAGE LPITS}

A laboratory system for the performance evaluation of LPVTs with analog and digital output by comparison with a reference transducer is developed (Fig.9). The system includes a generation and measurement section and is based on a National Instruments (NI) PCI eXtension for Instrumentation (PXI) platform. As to the generation section, low voltage (LV) test waveforms are generated by an Arbitrary Waveform Generator

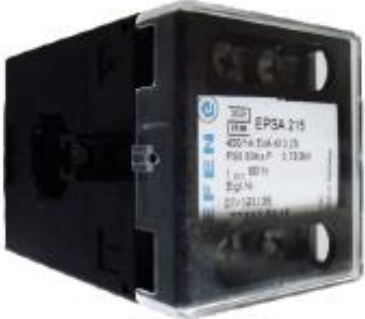

(a) Class $0.2 \mathrm{~S} 400 \mathrm{~A} / 1 \mathrm{~A}$.

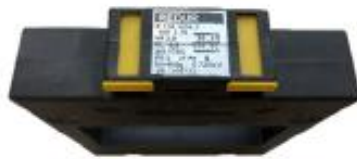

(b) Class $0.5500 \mathrm{~A} / 1 \mathrm{~A}$.

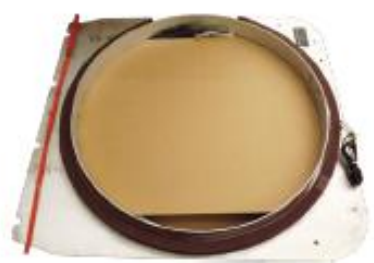

(c) Class $15000 \mathrm{~A} / 5 \mathrm{~A}$.

Fig. 5: Photos of the inductive current transformers.

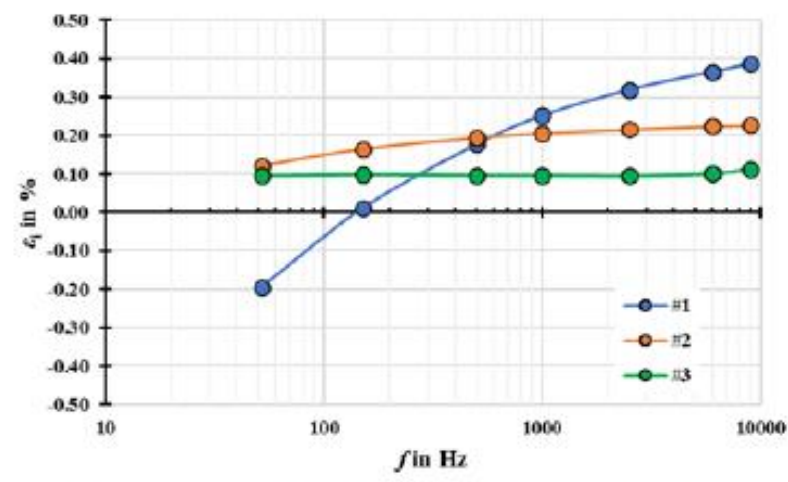

(a) Ratio errors. \#1 Class $0.2 \mathrm{~S}$, \#2 Class 0.5 , \#3 Class 1.

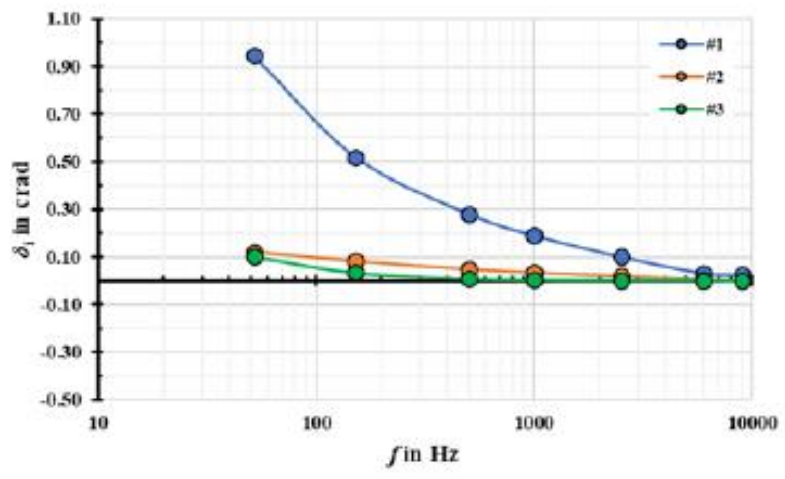

(b) Phase errors. \#1 Class $0.2 \mathrm{~S}$, \#2 Class 0.5 , \#3 Class 1.

Fig. 6: Measurement results of inductive current transformers.

This is an author-created, un-copyedited version of an article accepted for publication in 2021 IEEE 11th International Workshop on Applied Measurements for Power Systems (AMPS), DOI: 10.1109/AMPS50177.2021.9586040. The presented work is used in the project 17IND06 (FutureGrid II) which have received funding from the EMPIR programme co-financed by the Participating States and from the European Union's Horizon 2020 research and innovation programme. 


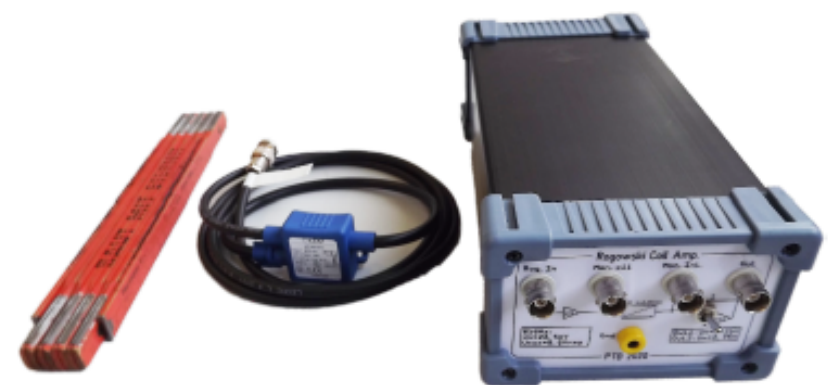

Fig. 7: Rogowski coils with the corresponding electronic intergrator.

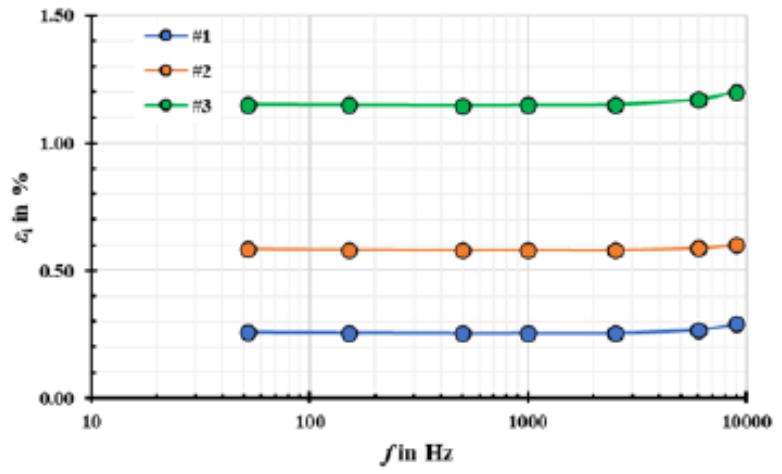

(a) Ratio errors. \#1 $\Phi=70 \mathrm{~mm}$,\#2 $\Phi=126 \mathrm{~mm}$, $\# 3 \Phi=175 \mathrm{~mm}$.

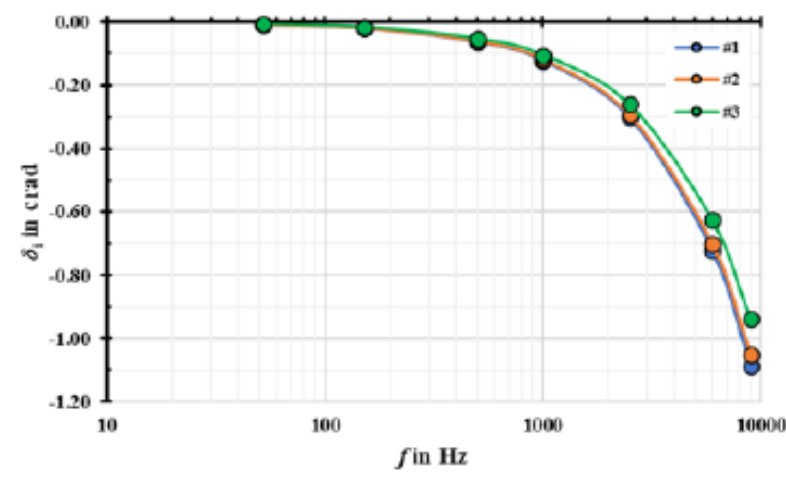

(b) Phase errors. \#1 $\Phi=70 \mathrm{~mm}$, \#2 $\Phi=126 \mathrm{~mm}$, \#3 $\Phi=175 \mathrm{~mm}$.

Fig. 8: Measurement results of three Rogowski coils.

(AWG) NI 5421, (16 bit, $\pm 12 \mathrm{~V}, 100 \mathrm{MHz}$ maximum sampling rate, $256 \mathrm{MB}$ on-board memory).

The LV waveforms are then amplified by a Trek high-voltage wideband power amplifier $\left(30 \mathrm{kV}_{p k}, 20 \mathrm{~mA}_{p k}\right)$ wide bandwidth (from DC to $2.5 \mathrm{kHz}$ at full voltage and $30 \mathrm{kHz}$ at reduced voltages), high slew rate $(<550 \mathrm{~V} / \mu \mathrm{s})$ and low noise.

The $10 \mathrm{MHz}$ PXI clock is used as a reference clock for AWG Phase Locked Loop (PLL) circuitry and, by means of a second AWG, it can also be used to obtain the master time-base clock of the comparator device (which needs a time-base clock of $12.8 \mathrm{MHz}$ ) in the measurement section. The $10 \mathrm{MHz}$ PXI clock can be disciplined with an accuracy of $10 \mathrm{~ns}$ through
TABLE I: Measurement results of current channel L1 of a Siemens SAMU Siprotec 5.

\begin{tabular}{cccc}
\hline Primary current (A) & Test point $(\%)$ & $\varepsilon(\%)$ & $\Delta \varphi$ (crad) \\
\hline 3995 & 400 & -0.06 & 0.19 \\
1998 & 200 & -0.08 & 0.25 \\
1199 & 120 & -0.13 & 0.29 \\
993 & 99 & -0.14 & 0.30 \\
799 & 80 & -0.16 & 0.31 \\
500 & 50 & -0.20 & 0.33 \\
199 & 20 & -0.25 & 0.37 \\
50 & 5 & -0.27 & 0.37 \\
20 & 2 & -0.28 & 0.36 \\
10 & 1 & -0.31 & 0.39 \\
\hline
\end{tabular}

the NI $6683 \mathrm{H}$ timing and synchronization module. It can be free running or synchronized to PTP IEEE 1588, or 1 Pulse per Second (PPS).

The synchronization signals (i.e. PTP or 1 PPS) are obtained through a IEEE 1588 PTP Grand Master clock synchronized to absolute time via GPS. It can be also used to provide 1 PPS or PTP signal to the device under test when a LPVT with digital output (DLPVT) is calibrated.

The generated voltage is measured by a $30 \mathrm{kV}$ wideband resistive-capacitive reference divider built and characterized at INRIM [13].

The measured reference and the test signals, when a LPVT with analogue output is calibrated, are acquired by a comparator that includes a NI compact Data AcQuisition system (cDAQ) with various acquisition modules (input range from $\pm 0.5 \mathrm{~V}$ to $\pm 425 \mathrm{~V}, 24$ bit, $50 \mathrm{kHz}$ ).

The software for data processing and instrument control is developed in LabVIEW. A large variety of signals can be generated, such as sinusoidal, fundamental plus one or $\mathrm{N}$ harmonics, amplitude and phase modulated signals, frequency ramps, PQ events reconstructed from available database of on-site recorded signal or user simulated.

Extension to higher voltages, can be obtained by a characterised step-up transformer $(220 \mathrm{~V} / 200 \mathrm{kV})$ with low total harmonic distortion (THD), e.g. less than some parts in $10^{3}$ in the generation section. As reference sensor, the use of a high voltage capacitive divider is presently under study.

\section{MEASUREMENT SCENARIO AND TEST DESCRIPTION}

As a first application, the possible errors introduced by a LPIT when used to reduce voltage/current upstream a PMU or a PQMU are estimated by the developed system. To this end the same voltage/current signal is applied to the reference transducer and the LPIT under test and their outputs are acquired by the comparator and processed by two identical and ideal devices, simulated by software, which implement PQMUs or PMUs algorithms.

\section{A. Test description}

As a first example of application of the developed generation and measurement setups, performances of LPITs are assessed

This is an author-created, un-copyedited version of an article accepted for publication in 2021 IEEE 11th International Workshop on Applied Measurements for Power Systems (AMPS), DOI: 10.1109/AMPS50177.2021.9586040. The presented work is used in the project 17IND06 (FutureGrid II) which have received funding from the EMPIR programme co-financed by the Participating States and from the European Union's Horizon 2020 research and innovation programme. 


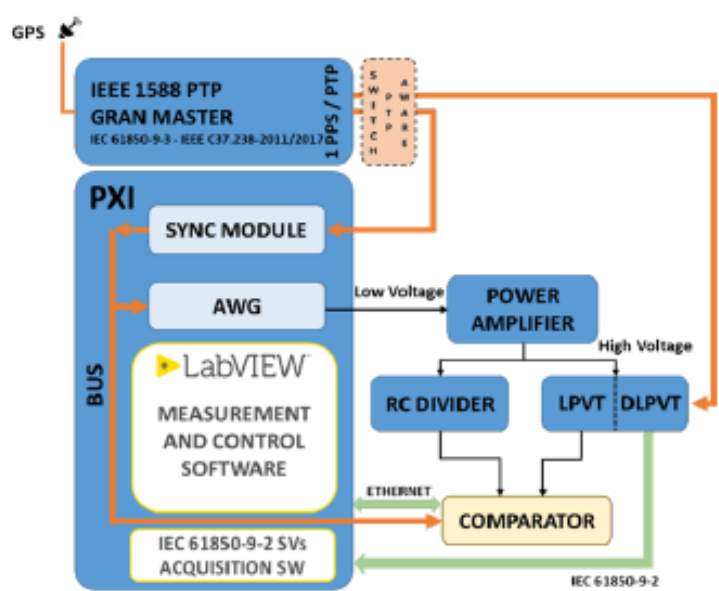

Fig. 9: Block diagram of the laboratory reference system for LPVT/DLPVT calibration.

under different conditions. More specifically, two test waveforms are here selected to evaluate the behavior of LPVTs when they are used to sense and reduce a steady-state (A) and a dynamic (B) voltage signal.

1) Fundamental plus $N$ Harmonic tones (FHN): The considered steady state voltage waveform consists in a multitoned signal composed of a fundamental tone at power frequency and $\mathrm{N}$ superimposed harmonic tones. The mathematical description is:

$$
v_{F H N}(t)=\sqrt{2} U_{r} \sin \left(2 \pi f_{0} t\right)+\sqrt{2} \sum_{h=2}^{N} U_{h} \sin \left(2 \pi h f_{0} t+\varphi_{h}\right)
$$

where:

- $U_{r}$ is the rms value of the rated primary voltage

- $f_{0}$ is the power frequency

- $U_{h}$ is the rms value of the h-harmonic order tone

- $\varphi_{h}$ is the phase delay between the fundamental tone and the h-harmonic order tone

Harmonic tone amplitudes $\left(U_{h}\right)$ are fixed to be compliant with medium voltage power grid harmonic limits as indicated by the standard [14] while the phases $\left(\varphi_{h}\right)$ are random selected.

For each generated frequency tone, both ratio and phase errors are evaluated according to Eq.5 and Eq.6:

$$
\begin{gathered}
\varepsilon_{h}=\frac{k_{r} U_{s, h}-U_{p, h}}{U_{p, h}} \\
\varphi_{h}=\varphi_{s, h}-\varphi_{p, h}
\end{gathered}
$$

where:

- $k_{r}=U_{p, r} / U_{s, r}$ is the rated scale factor SF ( $U_{p, r}$ and $U_{s, r}$ are the rated primary and secondary voltages);

- $U_{p, h}$ and $U_{s, h}$ are the rms values of the primary and secondary h-order harmonic voltage;

- $\varphi_{p, h}$ and $\varphi_{s, h}$ are phase angles of the primary and secondary h-order harmonic voltage

In addition, the THD value is also evaluated.
2) Frequency ramp (FR): The performances of the sensors are evaluated under a Frequency Ramp (FR) test for PMU dynamic compliance verification [5]. The applied waveform is generated according to:

$$
v_{F R}(t)=\sqrt{2} U_{r} \cos \left(2 \pi f_{0} t+\pi R_{f} t^{2}\right)
$$

where $U_{r}$ and $f_{0}$ are the rms voltage and the rated frequency, while $R_{f}$ is the frequency ramp rate.

The fundamental primary and secondary synchrophasors $V_{p}$ and $V_{s}$ are estimated via the interpolated discrete Fourier transform on an observation interval of four cycles of the fundamental frequency (i.e. $0.08 \mathrm{~s}$ ) with a reporting rate of $50 \mathrm{~Hz}$. Starting from them, the interest indices (from Eq. 8 to Eq.12) defined as in [5] are evaluated: ratio error, phase error, Total Vector Error (TVE), the Frequency Error (FE) and the rate of change of frequency (ROCOF) error (RFE).

$$
\begin{gathered}
\varepsilon=100 \frac{k_{r}\left|V_{s}\right|-\left|V_{p}\right|}{|V|_{p}} \\
\Delta \varphi=\angle V_{s}-\angle V_{p}
\end{gathered}
$$

$$
\begin{gathered}
T V E=\sqrt{\frac{\left(\operatorname{Re}\left(k_{r} V_{s}\right)-\operatorname{Re}\left(V_{p}\right)\right)^{2}+\left(\operatorname{Im}\left(k_{r} V_{s}\right)-\operatorname{Im}\left(V_{p}\right)\right)^{2}}{\operatorname{Re}\left(V_{p}\right)^{2}+\operatorname{Im}\left(V_{p}\right)^{2}}} \\
F E=f_{0, s}-f_{0, p} \\
R F E=\frac{d f_{0, s}}{d t}-\frac{d f_{0, m}}{d t}=\text { ROCOF }_{s}-\text { ROCOF }_{p}
\end{gathered}
$$

\section{EXPERIMENTAL RESULTS}

The performances of two LPVTs are investigated. The first device under test, here named $L P V T_{A}$, is a commercial gasinsulated divider with the following features:

- measuring range $\pm 45 \mathrm{kV}$;

- rated SF $10000 \mathrm{~V} / \mathrm{V}$;

- $0.2 \%$ rated accuracy at $50 / 60 \mathrm{~Hz}$

- $1 \mathrm{MHz}$ bandwidth.

The second device under test $\left(L P V T_{B}\right)$ is a portable precision high voltage divider for DC and AC measurement:

- measuring range $\pm 30 \mathrm{kV}$;

- rated scale factor $1000 \mathrm{~V} / \mathrm{V}$;

- rated accuracy $0.1 \%$ at $50 / 60 \mathrm{~Hz}$.

In the following, both FHN and FR results are shown after testing the two LPVTs.

\section{A. FHN results}

For the considered test the fundamental tone is set at $U_{n}=$ $13 \mathrm{kV}$ and $f_{0}=50 \mathrm{~Hz}$, nine superimposed tones are selected. Time behaviour is shown in Fig.10. The multitoned generated signal has THD equal to $7.73 \%$, slightly lower than the THD limit indicated in [14], i.e. $8 \%$. For each generated frequency, ratio and phase errors are evaluated according to Eq.5 and Eq.6. The THD measured by the $L P V T_{A}$ is equal to $7.81 \%$ whereas the THD value measured by the $L P V T_{B}$ is equal to

This is an author-created, un-copyedited version of an article accepted for publication in 2021 IEEE 11th International Workshop on Applied Measurements for Power Systems (AMPS), DOI: 10.1109/AMPS50177.2021.9586040. The presented work is used in the project 17IND06 (FutureGrid II) which have received funding from the EMPIR programme co-financed by the Participating States and from the European Union's Horizon 2020 research and innovation programme. 
$7.39 \%$. Thus, the $L P V T_{B}$ introduces a deviation of $0.34 \%$ in the THD measurement and this is essentially due to an unsuitable frequency behaviour. In fact, as can be seen in Table II, $L P V T_{B}$ does not fulfil the phase error $(\Delta \varphi)$ accuracy requirements for $0.1 \%$ class device [10] and it respect the limit for the ratio error $(\varepsilon)$ only for the first 6 harmonics.

TABLE II: $L P V T_{B}$ FHN Test Parameters and Results.

\begin{tabular}{ccccc}
\hline $\begin{array}{c}\text { Frequency } \\
(\mathrm{Hz})\end{array}$ & $\begin{array}{c}\text { Amplitude } \\
\text { (\% rated) }\end{array}$ & $\begin{array}{c}\Delta \varphi_{h}-\Delta \varphi_{f} \\
(\mathrm{rad})\end{array}$ & $\begin{array}{c}\varepsilon \\
(\%)\end{array}$ & $\begin{array}{c}\Delta \varphi \\
(\mathrm{mrad})\end{array}$ \\
\hline 50 & 100 & 0 & 0.029 & -22.39 \\
100 & 2 & 2.33 & -0.16 & -44.74 \\
150 & 5 & -1.65 & -0.45 & -66.24 \\
200 & 1 & 0.67 & -0.83 & -88.21 \\
300 & 0.5 & -0.98 & -1.88 & -130.53 \\
450 & 1.5 & -0.32 & -4.15 & -191.11 \\
550 & 3.5 & -1.97 & -6.00 & -229.13 \\
650 & 3 & -2.23 & -8.11 & -264.79 \\
850 & 2 & -0.64 & -12.89 & -328.37 \\
1150 & 1.5 & 0.69 & -20.82 & -401.89 \\
\hline
\end{tabular}

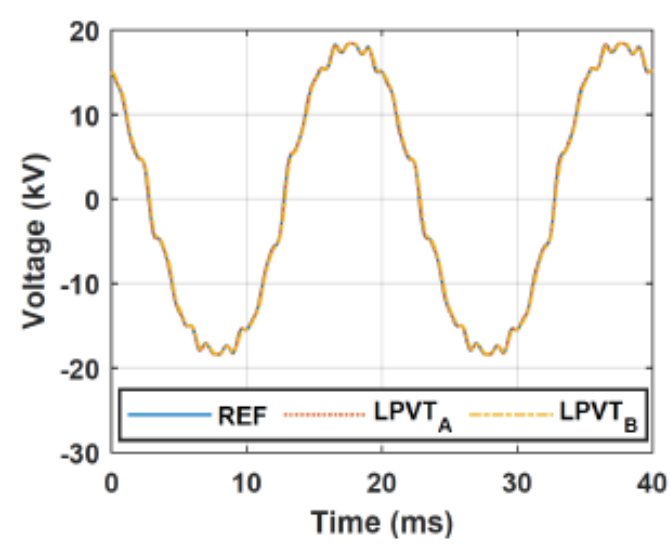

Fig. 10: FHN test time domain signal.

\section{B. FR results}

The frequency ramp rms voltage amplitude is set equal to $13 \mathrm{kV}, f_{0}$ is fixed at $50 \mathrm{~Hz}$, two values of $R_{f}$ are tested, i.e. $\pm 1 \mathrm{~Hz} / \mathrm{s}$ and the investigated ramp range $\Delta f$ is $5 \mathrm{~Hz}$, thus, for $R_{f}=1 \mathrm{~Hz} / \mathrm{s}$ the frequency ramp goes from $50 \mathrm{~Hz}$ up to $55 \mathrm{~Hz}$ while for $R_{f}=-1 \mathrm{~Hz} / \mathrm{s}$ from $50 \mathrm{~Hz}$ to $45 \mathrm{~Hz}$. For sake of brevity only results related to $R_{f}=+1 \mathrm{~Hz} / \mathrm{s}$ are reported and they are shown in Table III where also the limits stated for the M class PMUs are provided [5].

As regard the FE and RFE, mean values for both LPVTs, are far below the limits required by [5]. As the TVE, the mean value of $L P V T_{A}$ cannot be considered negligible (about $50 \%$ ) with respect to the maximum error prescribed for the $\mathrm{M}$ class PMU while the TVE of the $L P V T_{B}$ is outright twice as much the value imposed for M class PMU by [5].

\section{CONCLUSIONS}

This paper shows the work carried out within the EMPIR "Future Grid II" project. Two different calibration systems
TABLE III: FR Test Results.

\begin{tabular}{cccc}
\hline Meas. Quantity & $L P V T_{A}$ & $L P V T_{B}$ & M-class PMU limits \\
\hline$\varepsilon(\%)$ & 0.025 & 0.021 & N/A \\
$\Delta \varphi$ (mrad) & 3.85 & -23.73 & N/A \\
TVE (\%) & 0.47 & 2.33 & 1 \\
FE (mHz) & 0.0094 & -0.066 & 5 \\
RFE (mHz/s) & 0.063 & 0.069 & 100 \\
\hline
\end{tabular}

for current and voltage LPITs have been presented, allowing technicians to calibrate the DUTs up to $400 / \sqrt{3} \mathrm{kV}$ for voltage LPIT and $2 \mathrm{kA}$ for current LPIT with complex test waveforms to emulate real-life conditions, such as steady state, dynamic or temporary events. These laboratory calibration setups make possible to provide traceability to the tested equipments with a basic uncertainty in the order of $30 \mathrm{ppm}$ and $50 \mu \mathrm{rad}$ for ratio and phase errors respectively. Applications of the systems to LPITs calibration under steady-state and dynamic waveforms are presented. The results obtained show the capability of the systems to faithfully reproduce PQ events and test waveforms in general.

\section{REFERENCES}

[1] IEC 61000-4-30:2015 - Electromagnetic compatibility (EMC) - Part 4-30: Testing and measurement techniques - Power quality measurement methods, 2015.

[2] IEC 61000-4-7:2002 - Electromagnetic compatibility (EMC) - Part 4-7 Testing and measurement techniques - General guide on harmonics and interharmonics measurements and instrumentation, for power supply systems and equipment connected thereto.

[3] IEC 62586-2:2013 - Power quality measurement in power supply systems - Part 2: Functional tests and uncertainty requirements, 2013.

[4] IEC 62586-1:2013 - Power quality measurement in power supply systems - Part 1: Power quality instruments (PQI), 2013.

[5] IEEE and IEC, IEEE 60255-118-1 - Measuring Relays and Protection Equipment - Part 118-1: Synchrophasor for Power System - Measurements, 2018.

[6] R. Stiegler, J. Meyer, J. Kilter, and S. Konzelmann, "Assessment of voltage instrument transformers accuracy for harmonic measurements in transmission systems," in Proceedings of International Conference on Harmonics and Quality of Power, ICHQP, 2016, pp. 152-157.

[7] G. Crotti, D. Gallo, D. Giordano, C. Landi, M. Luiso, and M. Modarres, "Frequency Response of MV Voltage Transformer Under Actual Waveforms," IEEE Transactions on Instrumentation and Measurement, vol. 66, no. 6, pp. 1146-1154, 2017. [Online]. Available: http://ieeexplore.ieee.org/document/7842615/

[8] G. D'Avanzo, A. Delle Femine, D. Gallo, C. Landi, and M. Luiso, "Impact of inductive current transformers on synchrophasor measurement in presence of modulations," Measurement, vol. 155, 2020.

[9] IEC 60044-8:2002 Instrument transformers - Part 8: Electronic current transformers, 2002.

[10] IEC, IEC 61869-6:2016 - Instrument Transformers Part 6: Additional General Requirements for Low Power Instrument Transformers, 2016.

[11] _- "IEC 61850-9-2:2011 - Communication networks and systems for power utility automation - Part 9-2: Specific communication service mapping (SCSM) - Sampled values over ISO/IEC 8802-3," 2011.

[12] Y. Chen, E. Mohns, M. Seckelmann, and S. De Rose, "Traceable calibration system for non-conventional current sensors with analogue or digital output," IEEE 11th International Workshop on Applied Measurements for Power Systems (AMPS) (submitted), 2021.

[13] G. Crotti, D. Gallo, D. Giordano, C. Landi, M. Luiso, M. Modarres, and M. Zucca, "Frequency Compliance of MV Voltage Sensors for Smart Grid Application," IEEE Sensors Journal, vol. 17, no. 23, pp. 7621-7629, 2017.

[14] EN 50160:2010 - Voltage characteristics of electricity supplied by public distribution networks.

This is an author-created, un-copyedited version of an article accepted for publication in 2021 IEEE 11th International Workshop on Applied Measurements for Power Systems (AMPS), DOI: 10.1109/AMPS50177.2021.9586040. The presented work is used in the project 17IND06 (FutureGrid II) which have received funding from the EMPIR programme co-financed by the Participating States and from the European Union's Horizon 2020 research and innovation programme. 